\title{
An extension of some variant of Meijer type integrals in the class of Boehmians
}

\section{Praveen Agarwal ${ }^{1}$, Shrideh KQ Al-Omari ${ }^{2}$ and Jaekeun Park ${ }^{3 *}$}

\section{"Correspondence:}

jkpark@hanseo.ac.kr

${ }^{3}$ Department of Mathematics, Hanseo University, Chungnam-do, Seosan-si, 356-706, Republic of Korea

Full list of author information is available at the end of the article

\begin{abstract}
In this paper we establish certain spaces of Boehmians for some Meijer type integral transform. The extended Meijer type integral transform is defined and some desired properties are obtained in the class of Boehmians. Further theorems are also discussed with some details.
\end{abstract}

Keywords: $\mathbf{1}_{\alpha_{1}, \alpha_{2}}$ transform; generalized function; Meijer-type integral; Krätzel transform; Boehmian space

\section{Introduction}

During the past decades, a number of Meijer-type integral transforms have been studied by many authors such as Meijer [1], Pandey [2], Srivastava [3], Srivastava and Vyas [4] and many others. Some variants of Meijer-type integrals were given by Krätzel [5-8] and, recently, by Waphare [9]. In 1990, a new version of Meijer-type integral, referred to as an $M_{\alpha, \beta}$-integral, has been investigated and its inversion formula was duly achieved by [10]. This variant of this transform was formed to generalize those obtained by Krätzel [6], Conlan and Koh [11], and Rodriguez [12] as well.

In this article, we consider some variant defined, under conditions of regularity and convergence, by Betancor [13] as

$$
\boldsymbol{\Phi}(\gamma)=\mathbf{l}_{\alpha_{1}, \alpha_{2}}(\phi(\zeta))(\gamma)=\gamma^{-\alpha_{2}} \int_{0}^{\infty} \zeta^{\alpha_{1}, \alpha_{2}-1} \mathbf{l}_{\alpha_{1}-1}(\gamma \zeta) \phi(\zeta) d \zeta
$$

with the inversion formula given as

$$
\mathbf{1}_{\alpha_{1}, \alpha_{2}}^{-1}(\phi(\gamma))(\zeta)=\frac{\zeta^{-\alpha_{2}}}{i \pi} \int_{\Lambda_{c}} \gamma^{\alpha_{1}, \alpha_{2}-1} \boldsymbol{\epsilon}_{\alpha_{1}-1}(\gamma \zeta) \boldsymbol{\Phi}(\gamma) d \gamma
$$

where $\Lambda_{c}=\{w \in \mathbb{C}: \operatorname{Re} \sqrt{2 w}=c>0\}, \alpha_{1}, \alpha_{2} \in \mathbb{C}, \mathbf{l}_{v}$ and $\boldsymbol{\epsilon}_{v}$ are the modified Bessel-Clifford functions of the third and first kind of order $v$, respectively.

The modified Bessel functions $\boldsymbol{\epsilon}_{v}$ and $\mathbf{l}_{v}$ are indeed related to the homologous Bessel functions $\mathbf{j}_{v}$ and $\mathbf{k}_{v}$ by the formulas

$$
\boldsymbol{\epsilon}_{v}(x)=x^{\frac{-v}{2}} \mathbf{j}_{v}(2 \sqrt{x})
$$

(c) 2016 Agarwal et al. This article is distributed under the terms of the Creative Commons Attribution 4.0 International License (http://creativecommons.org/licenses/by/4.0/), which permits unrestricted use, distribution, and reproduction in any medium, provided you give appropriate credit to the original author(s) and the source, provide a link to the Creative Commons license, and indicate if changes were made. 
and

$$
\mathbf{l}_{v}(x)=x^{\frac{-v}{2}} \mathbf{k}_{v}(2 \sqrt{x})
$$

which are solutions of the differential equation $x y^{\prime \prime}+(v+1) y^{\prime}-y=0$. They also satisfy the relations $\mathbf{l}_{v}^{(n)}=(-1)^{n} \mathbf{I}_{v+n}$ and $\boldsymbol{\epsilon}_{v}^{(n)}=\boldsymbol{\epsilon}_{v+n}$, for every choice of $n \in \mathbb{N}$.

In addition, the integral representation of the modified Bessel function $\mathbf{l}_{\alpha-1}$ was introduced by Rodriguez [10], Meijer [1] and Waphare [9] as

$$
\mathbf{1}_{\alpha-1}(\gamma \zeta)=\frac{1}{2} \int_{0}^{\infty} \tau^{-\alpha} e^{-\tau-\frac{\gamma \zeta}{\tau}} d \tau
$$

For a complete account of $\mathbf{1}_{\alpha-1}$ and $\boldsymbol{\epsilon}_{\alpha-1}$ functions we refer to Rodriguez in [10] and Betancor in [13].

The class of Boehmians was introduced to generalize regular operators [14]. The minimal structure necessary for the abstract construction of Boehmian spaces consists of the following elements:

(i) A nonempty set $\mathbf{a}$.

(ii) A commutative semigroup (b, $*$ ).

(iii) An operation $\odot: \mathbf{a} \times \mathbf{b} \rightarrow \mathbf{a}$ such that for each $x \in \mathbf{a}$, the following formula is satisfied:

$$
x \odot\left(\delta_{1} * \delta_{2}\right)=\left(x \odot \delta_{1}\right) \odot \delta_{2}
$$

for every choice of $\delta_{1}, \delta_{2} \in \mathbf{b}$.

(iv) A collection $\Delta \subset \mathbf{b}$ such that:

(a) if $x, y \in \mathbf{a},\left(\delta_{n}\right) \in \Delta, x \odot \delta_{n}=y \odot \delta_{n}$ for all $n$, then $x=y$;

(b) if $\left(\delta_{n}\right),\left(\psi_{n}\right) \in \Delta$, then $\left(\delta_{n} * \psi_{n}\right) \in \Delta$.

The elements of $\Delta$ are called delta sequences. Denote by $g$ the set

$$
g=\left\{\left(x_{n}, \delta_{n}\right): x_{n} \in \mathbf{a},\left(\delta_{n}\right) \in \Delta, x_{n} \odot \delta_{m}=x_{m} \odot \delta_{n}, \forall m, n \in \mathbb{N}\right\} .
$$

Then, if $\left(x_{n}, \delta_{n}\right),\left(y_{n}, \psi_{n}\right) \in g, x_{n} \odot \psi_{m}=y_{m} \odot \delta_{n}, \forall m, n \in \mathbb{N}$, then we say $\left(x_{n}, \delta_{n}\right) \sim\left(y_{n}, \psi_{n}\right)$. The relation $\sim$ is an equivalence relation in $g$. The space of equivalence classes ing is denoted by $\boldsymbol{\alpha}$. Elements of $\boldsymbol{\alpha}$ are called Boehmians. Between a and $\boldsymbol{\alpha}$ there is a canonical embedding expressed as

$$
x \rightarrow \frac{x \odot \delta_{n}}{\delta_{n}}
$$

The operation $\odot$ can be extended to $\boldsymbol{\alpha} \times \mathbf{a}$ by

$$
\frac{x_{n}}{\delta_{n}} \odot t=\frac{x_{n} \odot t}{\delta_{n}} .
$$

In $\alpha$, we have two types of convergence.

$\delta$-convergence: A sequence $\left(\beta_{n}\right)$ in $\boldsymbol{\alpha}$ is said to be $\delta$-convergent to $\beta$ in $\boldsymbol{\alpha}$, denoted by $\beta_{n} \stackrel{\delta}{\rightarrow} \beta$, if there exists a delta sequence $\left(\delta_{n}\right)$ such that $\left(\beta_{n} \odot \delta_{n}\right),\left(\beta \odot \delta_{n}\right) \in \mathbf{a}, \forall k, n \in \mathbb{N}$, and $\left(\beta_{n} \odot \delta_{k}\right) \rightarrow\left(\beta \odot \delta_{k}\right)$ as $n \rightarrow \infty$, in a, for every $k \in \mathbb{N}$. 
$\Delta$-convergence: A sequence $\left(\beta_{n}\right)$ in $\alpha$ is said to be $\Delta$-convergent to $\beta$ in $\alpha$, denoted by $\beta_{n} \stackrel{\Delta}{\rightarrow} \beta$, if there exists a $\left(\delta_{n}\right) \in \Delta$ such that $\left(\beta_{n}-\beta\right) \odot \delta_{n} \in \mathbf{a}, \forall n \in \mathbb{N}$, and $\left(\beta_{n}-\beta\right) \odot \delta_{n} \rightarrow 0$ as $n \rightarrow \infty$ in a.

For the abstract construction of Boehmian spaces, we refer to Karunakaran and Roopkumar [15], Karunakaran and Vembu [16], Mikusinski [17], Al-Omari [18-20], Al-Omari and Kilicman [21], Nemzer [22], and some others.

\section{Some notations and necessary theorems}

Denote by $\Omega(0, \infty)$ the Schwartz' space of test functions of compact supports defined on $(0, \infty)$ and by $\digamma_{\text {loc }}((0, \infty))$, the set of all continuous functions of bounded supports over $(0, \infty)$ such that

$$
\int_{\mathrm{k}}|f(x)| d x
$$

is finite for every compact subset $\mathrm{k}$ of $(0, \infty)$.

The following are two definitions needed for our next investigation.

Definition 1 The Mellin-type convolution product of first kind is defined by Karunakaran and Roopkumar as [15]

$$
(\phi * \psi)(\zeta)=\int_{0}^{\infty} x^{-1} \phi\left(\zeta x^{-1}\right) \psi(x) d x
$$

The general properties of $*$ are given by

(i) $(\phi * \psi)(t)=(\psi * \phi)(t)$;

(ii) $((\phi+\psi) * \varphi)(t)=(\phi * \varphi)(t)+(\psi * \varphi)(t)$;

(iii) $(\alpha \phi * \psi)(t)=\alpha(\psi * \phi)(t), \alpha$ is complex number;

(iv) $((\phi * \psi) * \varphi)(t)=(\phi *(\psi * \varphi))(t)$.

Definition 2 Let $\mathbf{l}^{1}$ denote the space of those functions which are complex-valued Lebesgue and integrable on $(0, \infty)$. Between the two functions $\phi$ and $\psi$ in $\mathbf{l}^{1}$ we define an operation $\circledast$ in terms of the integral equation

$$
(\phi \circledast \psi)(\gamma)=\int_{0}^{\infty} x^{\alpha_{1}+\alpha_{2}-1} \phi(\gamma x) \psi(x) d x
$$

for every choice of $\alpha_{1}$ and $\alpha_{2}$.

The integrals (7) and (8) are clearly related by the following theorem.

Theorem 3 Let $\phi$ and $\psi$ be complex-valued integrable functions in $\mathbf{1}^{1}$ and $\gamma \in(0, \infty)$. Then we have

$$
\mathbf{l}_{\alpha_{1}, \alpha_{2}}(\phi * \psi)(\gamma)=\left(\mathbf{l}_{\alpha_{1}, \alpha_{2}} \phi \circledast \psi\right)(\gamma),
$$

where $\alpha_{1}, \alpha_{2} \in \mathbb{C}$. 
Proof Let the hypothesis of the theorem be satisfied by some functions $\phi$ and $\psi$ in $\mathbf{l}^{1}$. Then by invoking (1) in (7) we get

$$
\begin{aligned}
\mathbf{1}_{\alpha_{1}, \alpha_{2}}(\phi * \psi)(\gamma) & =\gamma^{-\alpha_{2}} \int_{0}^{\infty} \zeta^{\alpha_{1}+\alpha_{2}-1} \mathbf{l}_{\alpha_{1}-1}(\gamma \zeta)(\phi * \psi)(\zeta) d \zeta \\
& =\gamma^{-\alpha_{2}} \int_{0}^{\infty} \zeta^{\alpha_{1}+\alpha_{2}-1} \mathbf{l}_{\alpha_{1}-1}(\gamma \zeta) \int_{0}^{\infty} x^{-1} \phi\left(\zeta x^{-1}\right) \psi(x) d x d \zeta \\
& =\gamma^{-\alpha_{2}} \int_{0}^{\infty} x^{-1} \psi(x) \int_{0}^{\infty} \zeta^{\alpha_{1}+\alpha_{2}-1} \mathbf{l}_{\alpha_{1}-1}(\gamma \zeta) \phi\left(\zeta x^{-1}\right) d x d \zeta
\end{aligned}
$$

By Fubini's theorem and setting variables, we obtain the integral equation

$$
\mathbf{1}_{\alpha_{1}, \alpha_{2}}(\phi * \psi)(\gamma)=\gamma^{-\alpha_{2}} \int_{0}^{\infty} \psi(x) x^{\alpha_{1}+\alpha_{2}-1} y^{\alpha_{1}+\alpha_{2}-1} \mathbf{l}_{\alpha_{1}-1}(\gamma(x y)) \phi(y) d y d x
$$

Hence, simplifications yield

$$
\begin{aligned}
\mathbf{1}_{\alpha_{1}, \alpha_{2}}(\phi * \psi)(\gamma)= & \int_{0}^{\infty} \psi(x) x^{\alpha_{1}+\alpha_{2}-1} \\
& \times\left((\gamma x)^{-\alpha_{2}} \int_{0}^{\infty} y^{\alpha_{1}+\alpha_{2}-1} \mathbf{l}_{\alpha_{1}-1}((\gamma x) y) \phi(y) d y\right) d x \\
= & \int_{0}^{\infty} x^{\alpha_{1}+\alpha_{2}-1} \mathbf{l}_{\alpha_{1}, \alpha_{2}} \phi(\gamma x) \psi(x) d x
\end{aligned}
$$

Therefore, by the aid of (8), we get

$$
\mathbf{1}_{\alpha_{1}, \alpha_{2}}(\phi * \psi)(\gamma)=\left(\mathbf{l}_{\alpha_{1}, \alpha_{2}} \phi \circledast \psi\right)(\gamma) .
$$

This completes the proof of the theorem.

Theorem 4 Let $\phi, \varphi$, and $\psi$ be integrable functions in $\mathbf{1}^{1}$ and $y \in(0, \infty)$. Then we have

$$
(\phi \circledast(\varphi * \psi))(y)=((\phi \circledast \varphi) \circledast \psi)(y) .
$$

Proof By taking into account (7) and (8) we by Fubini's theorem obtain

$$
\begin{aligned}
(\phi \circledast(\varphi * \psi))(y) & =\int_{0}^{\infty} \zeta^{\alpha_{1}+2 \alpha_{2}-1} \phi(y \zeta)(\varphi * \psi)(\zeta) d \zeta \\
& =\int_{0}^{\infty} \zeta^{\alpha_{1}+2 \alpha_{2}-1} \phi(y \zeta) \int_{0}^{\infty} x^{-1} \varphi\left(\zeta x^{-1}\right) \psi(x) d x d \zeta \\
& =\int_{0}^{\infty} x^{\alpha_{1}+2 \alpha_{2}-1} \psi(x) \int_{0}^{\infty} \phi(y x \tau) \varphi(z) \tau^{\alpha_{1}+2 \alpha_{2}-1} d \tau d x
\end{aligned}
$$

Setting variables gives

$$
(\phi \circledast(\varphi * \psi))(y)=\int_{0}^{\infty} x^{\alpha_{1}+2 \alpha_{2}-1}(\phi \circledast \varphi)(y x) \psi(x) d x .
$$


Hence, equation (8) reveals

$$
(\phi \circledast(\varphi * \psi))(y)=((\phi \circledast \varphi) \circledast \psi)(y) .
$$

This completes the proof of the theorem.

Theorem 5 Let $\phi \in \digamma_{\text {loc }}(0, \infty)$. Then $\mathbf{l}_{\alpha_{1}, \alpha_{2}}(\phi) \in \digamma_{\text {loc }}(0, \infty)$, for $\alpha_{1}<2, \alpha_{2} \neq 2-\alpha_{1}$.

Proof Let $\phi \in \digamma_{\text {loc }}(0, \infty)$ be arbitrary and $\mathrm{k}_{1}$ be a compact subset in $(0, \infty)$. Then, invoking (5) in (1) suggests

$$
\begin{aligned}
\int_{\mathrm{k}_{1}}\left|\mathbf{l}_{\alpha_{1}, \alpha_{2}}(\phi(\zeta))(\gamma)\right| d \gamma \leq & \int_{\mathrm{k}_{1}} \gamma^{-\alpha_{2}} \int_{0}^{\infty} \zeta^{\alpha_{1}+2 \alpha_{2}-1}\left|\mathbf{l}_{\alpha_{1}-1}(\gamma \zeta)\right||\phi(\zeta)| d \zeta d \gamma \\
\leq & \frac{1}{2} \int_{\mathrm{k}_{1}} \gamma^{-\alpha_{2}} \int_{0}^{\infty} \zeta^{\alpha_{1}+2 \alpha_{2}-1}\left|\int_{0}^{\infty} \tau^{-\alpha_{1}} e^{-\tau-\frac{\gamma \zeta}{\tau}} d \tau\right| \\
& \times|\phi(\zeta)| d \zeta d \gamma \\
= & \frac{1}{2} \int_{\mathrm{k}_{1}} \zeta^{\alpha_{1}+2 \alpha_{2}-1} \\
& \times\left|\int_{0}^{\infty}\left(\gamma \zeta y^{-1}\right)^{-\alpha_{1}} e^{-(\gamma \zeta)^{-1} y-y}(\gamma \zeta) y^{-2} d y\right||\phi(\zeta)| d \zeta d \gamma
\end{aligned}
$$

Setting variables reveals

$$
\begin{aligned}
\int_{\mathrm{k}_{1}}\left|\mathbf{l}_{\alpha_{1}, \alpha_{2}}(\phi(\zeta))(\gamma)\right| d \gamma \leq & \frac{1}{2} \int_{\mathrm{k}_{1}} \gamma^{-\alpha_{2}-\alpha_{1}+1} \int_{0}^{\infty} \zeta^{\alpha_{2}}\left|\int_{0}^{\infty} y^{\alpha_{1}-2} e^{-\left(\gamma \zeta y^{-1}\right)-y} d y\right| \\
& \times|\phi(\zeta)| d \zeta d \gamma
\end{aligned}
$$

By the fact that $e^{-\left(\gamma \zeta y^{-1}\right)-y}<e^{-y}$ we have

$$
\begin{aligned}
\int_{\mathrm{k}_{1}}\left|\mathbf{l}_{\alpha_{1}, \alpha_{2}}(\phi(\zeta))(\gamma)\right| d \gamma & \leq \frac{1}{2} \int_{\mathrm{k}_{1}} \gamma^{-\alpha_{2}-\alpha_{1}+1} \int_{0}^{\infty} \zeta^{\alpha_{2}}\left|\int_{0}^{\infty} y^{\alpha_{1}-2} e^{-y} d y\right||\phi(\zeta)| d \zeta d \gamma \\
& <\frac{1}{2} \int_{\mathrm{k}_{1}} \gamma^{-\alpha_{2}-\alpha_{1}+1} \int_{0}^{\infty} \zeta^{\alpha_{2}}\left(\int_{0}^{\infty} e^{-y} d y\right)|\phi(\zeta)| d \zeta d \gamma
\end{aligned}
$$

By the fact that $\left|y^{\alpha_{1}-2}\right|<1$, for $\alpha_{1}<1$, we get

$$
\int_{\mathrm{k}_{1}}\left|\mathbf{l}_{\alpha_{1}, \alpha_{2}}(\phi(\zeta))(\gamma)\right| d \gamma \leq \frac{1}{2} \int_{\mathrm{k}_{1}} \gamma^{-\alpha_{2}-\alpha_{1}+1} \int_{0}^{\infty} \zeta^{\alpha_{2}}|\phi(\zeta)| d \zeta d \gamma
$$

The last equation follows from the fact that $\int_{0}^{\infty} e^{-y} d y=1$.

Now, let $\mathrm{k}_{2}=[c, d], 0<c<d$, be a compact set containing the support of $\phi$, i.e., supp $\phi \subseteq$ $[c, d]$, and $\mathrm{k}_{1}=[a, b], 0<a<b$. Then we get

$$
\begin{aligned}
\int_{\mathrm{k}_{1}}\left|\mathbf{1}_{\alpha_{1}, \alpha_{2}}(\phi(\zeta))(\gamma)\right| d \gamma & <\frac{1}{2} \int_{a}^{b} \gamma^{-\alpha_{2}-\alpha_{1}+1}\left(\int_{c}^{d} \zeta^{\alpha_{2}}|\phi(\zeta)| d \zeta\right) d \eta \\
& <\frac{M}{2} \int_{a}^{b} \gamma^{-\alpha_{2}-\alpha_{1}+1} d \gamma
\end{aligned}
$$


where $M=\int_{c}^{d} \zeta^{\alpha_{2}}|\phi(\zeta)| d \zeta$. Hence,

$$
\begin{aligned}
\int_{\mathrm{k}_{1}}\left|\mathbf{1}_{\alpha_{1}, \alpha_{2}}(\phi(\zeta))(\gamma)\right| d \gamma & <\frac{M}{2} \frac{\left(b^{-\alpha_{2}-\alpha_{1}+2}-b^{-\alpha_{1}-\alpha_{1}+2}\right)}{-\alpha_{2}-\alpha_{1}+2} \\
& <\infty
\end{aligned}
$$

The theorem is therefore completely proved.

\section{Constructed spaces of Boehmians}

Here we begin by the following spaces of Boehmians, named $\boldsymbol{\alpha}\left(\digamma_{\text {loc }}, \Omega, *, *\right)$, and $\boldsymbol{\alpha}\left(\digamma_{\text {loc }}, \Omega\right.$, $*, \circledast)$, where $\Delta$ is a collection of delta sequence $\left(\delta_{n}\right)$ from $\Omega$ satisfying

$$
\begin{aligned}
& \Delta_{1}: \int_{0}^{\infty} \delta_{n}(x) d x=1 ; \\
& \Delta_{2}: \int_{0}^{\infty}\left|\delta_{n}(x)\right| d x<M, \quad M \in \mathbb{R}, M>0 ; \\
& \Delta_{3}: \operatorname{supp} \delta_{n}(x) \rightarrow 0 \quad \text { as } n \rightarrow \infty .
\end{aligned}
$$

Let us now establish that $\boldsymbol{\alpha}\left(\digamma_{\text {loc }}, \Omega, *, \circledast\right)$ is a Boehmian space. We prefer to omit the details of establishing $\boldsymbol{\alpha}\left(\digamma_{\text {loc }}, \Omega, *, *\right)$ since the construction of the latter is straightforward from the properties of $*$.

We prove certain theorems that are needed for this construction.

Theorem 6 Let $\phi \in \digamma_{\text {loc }}(0, \infty)$ and $\varphi, \psi \in \Omega(0, \infty)$. Then $\phi \circledast(\varphi * \psi)=(\phi \circledast \varphi) \circledast \psi$.

The proof of this theorem follows from Theorem 4. Details are thus omitted.

Theorem 7 Let $\phi \in \digamma_{\text {loc }}(0, \infty)$ and $\varphi \in \Omega(0, \infty)$. Then $\phi \circledast \varphi \in \digamma_{\text {loc }}(0, \infty)$.

Proof Let $\mathrm{k}_{1}$ be a compact subset in $(0, \infty)$. Then we have

$$
\begin{aligned}
\int_{\mathrm{k}_{1}}|(\phi \circledast \varphi)(\gamma)| d \gamma= & \int_{\mathrm{k}_{1}}\left|\int_{0}^{\infty} x^{\alpha_{1}+2 \alpha_{2}-1} \phi(\gamma x) \varphi(x) d x\right| d \gamma \\
\leq & \int_{\mathrm{k}_{1}} \int_{\mathrm{k}}\left|\int_{0}^{\infty} x^{\alpha_{1}+2 \alpha_{2}-1} \phi(\gamma x)\right||\varphi(x)| d x d \gamma \\
& \text { (by Fubini's theorem) } \\
\leq & \int_{\mathrm{k}}|\varphi(x)| \int_{\mathrm{k}_{1}}\left|x^{\alpha_{1}+2 \alpha_{2}-1} \phi(\gamma x)\right| d \gamma d x,
\end{aligned}
$$

where $\mathrm{k}$ is a compact set containing the $\operatorname{supp} \varphi$.

By $\Delta_{1}$, we obtain

$$
\int_{\mathrm{k}_{1}}|(\phi \circledast \varphi)(\gamma)| d \gamma \leq M M_{1},
$$

where $M_{1}=\int_{\mathrm{k}_{1}}\left|x^{\alpha_{1}+2 \alpha_{2}-1} \phi(\gamma x)\right| d \gamma$ and $M=\int_{\mathrm{k}}|\varphi(x)| d x$.

Hence, the theorem is completely proved. 
Theorem 8 Let $\phi_{1}, \phi_{2} \in \digamma_{\text {loc }}(0, \infty)$ and $\psi_{1}, \psi_{2} \in \Omega(0, \infty)$. Then the following hold:

(i) $\left(\phi_{1}+\phi_{2}\right) \circledast \psi_{1}=\phi_{1} \circledast \psi_{1}+\phi_{2} \circledast \psi_{1}$.

(ii) If $\phi_{n} \rightarrow \phi$ in $\digamma_{\text {loc }}(0, \infty)$ and $\psi \in \Omega(0, \infty)$, then

$$
\phi_{n} \circledast \psi \rightarrow \phi \circledast \psi \quad \text { as } n \rightarrow \infty .
$$

(iii) If $\left(\delta_{n}\right),\left(\psi_{n}\right) \in \Delta$ then $\left(\delta_{n} * \psi_{n}\right) \in \Delta$.

Proof The proof of parts (i) and (ii) follows from a simple integration. The proof of part (iii) follows from Roopkumar [23].

Hence, the theorem is proved.

Finally in this construction, we prove the following theorem.

Theorem 9 Let $\phi \in \digamma_{\mathrm{loc}}^{\mathrm{K}}(0, \infty)$ and $\left(\delta_{n}\right) \in \Delta$. Then $\phi \circledast \delta_{n} \rightarrow \phi$ as $n \rightarrow \infty$.

Proof Let k be a compact subset of $(0, \infty)$. Then, by $\Delta_{1}$, we write

$$
\begin{aligned}
\int_{\mathrm{k}}\left|\left(\phi \circledast \delta_{n}\right)(\gamma)-\phi(\gamma)\right| d \gamma & =\int_{\mathrm{k}}\left|\int_{0}^{\infty} x^{\alpha_{1}+2 \alpha_{2}-1} \phi(\gamma x) \delta_{n}(x) d x-\phi(\gamma)\right| d \gamma \\
& =\int_{\mathrm{k}}\left|\int_{0}^{\infty} x^{\alpha_{1}+2 \alpha_{2}-1} \phi(\gamma x) \delta_{n}(x) d x-\int_{0}^{\infty} \phi(\gamma) \delta_{n}(x) d x\right| d \gamma \\
& \leq \int_{\mathrm{k}} \int_{0}^{\infty}\left|x^{\alpha_{1}+2 \alpha_{2}-1} \phi(\gamma x)-\phi(\gamma)\right|\left|\delta_{n}(x)\right| d x d \gamma .
\end{aligned}
$$

Let $h(\gamma)=x^{\alpha_{1}+2 \alpha_{2}-1} \phi(\gamma x)-\phi(\gamma)$. Then $h(\gamma)$ is uniformly continuous for each $\gamma \in(0, \infty)$. Hence

$$
\int_{\mathrm{k}}\left|\left(\phi \circledast \delta_{n}\right)(\gamma)-\phi(\gamma)\right| d \gamma \rightarrow 0 \quad \text { as } n \rightarrow \infty .
$$

Therefore $\phi \circledast \delta_{n} \rightarrow \phi$ as $n \rightarrow \infty$.

This completes the proof of the theorem.

The space $\boldsymbol{\alpha}\left(\digamma_{\text {loc }}, \Omega, *, \circledast\right)$ is therefore constructed. Construction of the space $\boldsymbol{\alpha}\left(\digamma_{\text {loc }}, \Omega\right.$, $*, *)$ is almost similar.

We define the sum and multiplication by a scalar of two Boehmians in $\alpha\left(\digamma_{\text {loc }}, \Omega, *, \circledast\right)$ in the natural way by

$$
\left[\frac{\left(\phi_{n}\right)}{\left(\delta_{n}\right)}\right]+\left[\frac{\left(g_{n}\right)}{\left(\varphi_{n}\right)}\right]=\left[\frac{\left(\phi_{n}\right) \circledast\left(\varphi_{n}\right)+\left(g_{n}\right) \circledast\left(\delta_{n}\right)}{\left(\delta_{n}\right) *\left(\varphi_{n}\right)}\right]
$$

and $\rho\left[\frac{\left(\phi_{n}\right)}{\left(\delta_{n}\right)}\right]=\left[\frac{\left(\rho \phi_{n}\right)}{\left(\delta_{n}\right)}\right], \rho$ is a complex number.

Between $\digamma_{\text {loc }}$ and $\boldsymbol{\alpha}\left(\digamma_{\text {loc }}, \Omega, *, \circledast\right)$ there is a canonical embedding expressed as

$$
x \rightarrow \frac{x \circledast \delta_{n}}{\delta_{n}} .
$$


The operation $\circledast$ can be extended to $\boldsymbol{\alpha}\left(\digamma_{\text {loc }}, \Omega, *, \circledast\right) \times \digamma_{\text {loc }}$ by

$$
\frac{x_{n}}{\delta_{n}} \circledast t=\frac{x_{n} \circledast t}{\delta_{n}} .
$$

In $\boldsymbol{\alpha}\left(\digamma_{\text {loc }}, \Omega, *, \circledast\right)$, we have two types of convergence.

$\delta$-convergence: A sequence $\left(\beta_{n}\right)$ in $\boldsymbol{\alpha}\left(\digamma_{\text {loc }}, \Omega, *, \circledast\right)$ is said to be $\delta$-convergent to $\beta$ in $\boldsymbol{\alpha}\left(\digamma_{\text {loc }}, \Omega, *, \circledast\right)$ if there can be a delta sequence $\left(\delta_{n}\right)$ such that $\left(\beta_{n} \circledast \delta_{n}\right),\left(\beta \circledast \delta_{n}\right) \in \digamma_{\text {loc }}$, $\forall k, n \in \mathbb{N}$, and $\left(\beta_{n} \circledast \delta_{k}\right) \rightarrow\left(\beta \circledast \delta_{k}\right)$ as $n \rightarrow \infty$, in $\digamma_{\text {loc }}$, for every $k \in \mathbb{N}$.

$\Delta$-convergence: A sequence $\left(\beta_{n}\right)$ in $\boldsymbol{\alpha}\left(\digamma_{\text {loc }}, \Omega, *, \circledast\right)$ is said to be $\Delta$-convergent to $\beta$ in $\boldsymbol{\alpha}\left(\digamma_{\text {loc }}, \Omega, *, \circledast\right)$ if there can be a $\left(\delta_{n}\right) \in \Delta$ such that $\left(\beta_{n}-\beta\right) \circledast \delta_{n} \in \digamma_{\text {loc }}, \forall n \in \mathbb{N}$, and $\left(\beta_{n}-\right.$ $\beta) \circledast \delta_{n} \rightarrow 0$ as $n \rightarrow \infty$ in $\digamma_{\text {loc }}$.

\section{Introduction of the generalized Meijer-type integral}

Here we introduce a generalized Meijer-type integral. Then we show its properties in the space of Boehmians.

Definition 10 Let $\left[\frac{\left(\phi_{n}\right)}{\left(\delta_{n}\right)}\right] \in \boldsymbol{\alpha}\left(\digamma_{\text {loc }}, \Omega, *, *\right)$. Then we define the generalized Meijer-type integral transform of $\left[\frac{\left(\phi_{n}\right)}{\left(\delta_{n}\right)}\right]$ as $\mathbf{l}_{\alpha_{1}, \alpha_{2}}^{g e}\left[\frac{\left(\phi_{n}\right)}{\left(\delta_{n}\right)}\right]=\left[\frac{\mathbf{l}_{\alpha_{1}, \alpha_{2}\left(\phi_{n}\right)}}{\left(\delta_{n}\right)}\right]$ in $\boldsymbol{\alpha}\left(\digamma_{\text {loc }}^{\mathrm{K}}, \Omega, *, \circledast\right)$.

Theorem 5 shows that Definition 10 is well defined.

We discuss now some properties of $\mathbf{l}_{\alpha_{1}, \alpha_{2}}^{g e}$.

Theorem 11 Let $\beta_{1}, \beta_{2} \in \boldsymbol{\alpha}\left(\digamma_{\text {loc }}, \Omega, *, *\right)$. Then $\mathbf{l}_{\alpha_{1}, \alpha_{2}}^{g e}\left(\beta_{1} * \beta_{2}\right)=\mathbf{l}_{\alpha_{1}, \alpha_{2}}^{g e} \beta_{1} \circledast \beta_{2}$ in $\boldsymbol{\alpha}\left(\digamma_{\text {loc }}, \Omega\right.$, $*, \circledast)$.

Proof Assume the hypothesis of the theorem is satisfied for some $\beta_{1}, \beta_{2} \in \boldsymbol{\alpha}\left(\digamma_{\text {loc }}, \Omega, *, *\right)$. Therefore there are $\left(\phi_{n}\right),\left(\kappa_{n}\right) \in \digamma_{\text {loc }}(0, \infty)$ and $\left(\varphi_{n}\right),\left(\delta_{n}\right) \in \Delta$ such that $\beta_{1}=\left[\frac{\left(\phi_{n}\right)}{\left(\varphi_{n}\right)}\right]$ and $\beta_{2}=$ $\left[\frac{\left(\kappa_{n}\right)}{\left(\delta_{n}\right)}\right]$. Therefore,

$$
\mathbf{1}_{\alpha_{1}, \alpha_{2}}^{g e}\left(\beta_{1} * \beta_{2}\right)=\mathbf{l}_{\alpha_{1}, \alpha_{2}}^{g e}\left(\left[\frac{\left(\phi_{n}\right) *\left(\kappa_{n}\right)}{\left(\varphi_{n}\right) *\left(\delta_{n}\right)}\right]\right) .
$$

Definition 10 gives

$$
\mathbf{l}_{\alpha_{1}, \alpha_{2}}^{g e}\left(\beta_{1} * \beta_{2}\right)=\left[\frac{\mathbf{l}_{\alpha_{1}, \alpha_{2}}\left(\left(\phi_{n}\right) *\left(\kappa_{n}\right)\right)}{\left(\varphi_{n}\right) *\left(\delta_{n}\right)}\right]
$$

By the aid of Theorem 3 we obtain

$$
\mathbf{l}_{\alpha_{1}, \alpha_{2}}^{g e}\left(\beta_{1} * \beta_{2}\right)=\left[\frac{\left(\mathbf{l}_{\alpha_{1}, \alpha_{2}} \phi_{n}\right) \circledast\left(\kappa_{n}\right)}{\left(\varphi_{n}\right) \circledast\left(\delta_{n}\right)}\right] .
$$

On separating quotients this yields

$$
\mathbf{l}_{\alpha_{1}, \alpha_{2}}^{g e}\left(\beta_{1} * \beta_{2}\right)=\left[\frac{\left(\mathbf{l}_{\alpha_{1}, \alpha_{2}} \phi_{n}\right)}{\left(\varphi_{n}\right)}\right] \circledast\left[\frac{\left(\kappa_{n}\right)}{\left(\delta_{n}\right)}\right] .
$$

Hence, $\mathbf{l}_{\alpha_{1}, \alpha_{2}}^{g e}\left(\beta_{1} * \beta_{2}\right)=\mathbf{l}_{\alpha_{1}, \alpha_{2}}^{g e}\left(\beta_{1}\right) \circledast \beta_{2}$.

This completes the proof of the theorem. 


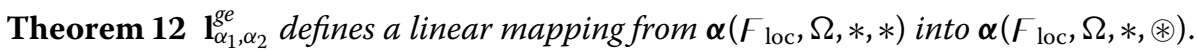

The proof is straightforward. Details are therefore omitted.

Theorem 13 Let $\left[\frac{\left(\phi_{n}\right)}{\left(\delta_{n}\right)}\right] \in \boldsymbol{\alpha}\left(\digamma_{\mathrm{loc}}, \Omega, *, *\right)$ and $\delta \in \Omega(0, \infty)$. Then we have

$$
\mathbf{l}_{\alpha_{1}, \alpha_{2}}^{g e}\left(\left[\frac{\left(\phi_{n}\right)}{\left(\delta_{n}\right)}\right] * \delta\right)=\left[\frac{\left(\mathbf{l}_{\alpha_{1}, \alpha_{2}} \phi_{n}\right)}{\left(\delta_{n}\right)}\right] \circledast \delta .
$$

Proof Let $\left[\frac{\left(\phi_{n}\right)}{\left(\delta_{n}\right)}\right] \in \boldsymbol{\alpha}\left(\digamma_{\text {loc }}, \Omega, *, *\right)$ and $\delta \in \Omega(0, \infty)$. By virtue of Definition 10 we write

$$
\mathbf{1}_{\alpha_{1}, \alpha_{2}}^{g e}\left(\left[\frac{\left(\phi_{n}\right)}{\left(\delta_{n}\right)}\right] * \delta\right)=\left[\frac{\mathbf{l}_{\alpha_{1}, \alpha_{2}}\left(\left(\phi_{n}\right) * \delta\right)}{\left(\delta_{n}\right)}\right] .
$$

Once again, Definition 10 and Theorem 3 give

$$
\mathbf{1}_{\alpha_{1}, \alpha_{2}}^{g e}\left(\left[\frac{\left(\phi_{n}\right)}{\left(\delta_{n}\right)}\right] * \delta\right)=\left[\frac{\left(\mathbf{l}_{\alpha_{1}, \alpha_{2}} \phi_{n}\right) \circledast \delta}{\left(\delta_{n}\right)}\right]=\left[\frac{\left(\mathbf{l}_{\alpha_{1}, \alpha_{2}} \phi_{n}\right)}{\left(\delta_{n}\right)}\right] \circledast \delta .
$$

This completes the proof of the theorem.

Theorem 14 The transform $\mathbf{l}_{\alpha_{1}, \alpha_{2}}^{\text {ge }}$ is consistent with $\mathbf{l}_{\alpha_{1}, \alpha_{2}}^{\text {ge }}: \digamma_{\text {loc }}(0, \infty) \rightarrow \digamma_{\text {loc }}(0, \infty)$.

Proof For every $\phi \in \digamma_{\text {loc }}(0, \infty)$, let $\beta \in \boldsymbol{\alpha}\left(\digamma_{\text {loc }}, \Omega, *, *\right)$ be the representative in the space $\boldsymbol{\alpha}\left(\digamma_{\text {loc }}, \Omega, *, *\right)$, then $\forall n \in \mathbb{N},\left(\delta_{n}\right) \in \Delta, \beta=\left[\frac{\phi *\left(\delta_{n}\right)}{\left(\delta_{n}\right)}\right]$. For all $n \in \mathbb{N}$ it is clear that $\left(\delta_{n}\right)$ is independent from the choice of the representative.

We have

$$
\mathbf{1}_{\alpha_{1}, \alpha_{2}}^{g e}(\beta)=\mathbf{l}_{\alpha_{1}, \alpha_{2}}^{g e}\left(\left[\frac{\phi *\left(\delta_{n}\right)}{\left(\delta_{n}\right)}\right]\right)=\left[\frac{\mathbf{l}_{\alpha_{1}, \alpha_{2}}\left(\phi *\left(\delta_{n}\right)\right)}{\left(\delta_{n}\right)}\right]=\left[\frac{\mathbf{l}_{\alpha_{1}, \alpha_{2}} \phi *\left(\delta_{n}\right)}{\left(\delta_{n}\right)}\right],
$$

which is the representative of $\mathbf{l}_{\alpha_{1}, \alpha_{2}}^{g e} \phi$ in the space $\digamma_{\text {loc }}(0, \infty)$.

Hence the proof is completed.

Theorem 15 The necessary and sufficient condition for $\left[\frac{\left(g_{n}\right)}{\left(\psi_{n}\right)}\right] \in \boldsymbol{\alpha}\left(\digamma_{\mathrm{loc}}, \Omega, *, \circledast\right)$ to be in the range of $\mathbf{1}_{\alpha_{1}, \alpha_{2}}^{g e}$ is that $g_{n}$ belongs to the range of $\mathbf{1}_{\alpha_{1}, \alpha_{2}}$ for every $n \in \mathbb{N}$.

Proof Let $\left[\frac{\left(g_{n}\right)}{\left(\psi_{n}\right)}\right]$ be in the range of $\mathbf{l}_{\alpha_{1}, \alpha_{2}}^{g e}$. Then of course $g_{n}$ belongs to the range of $\mathbf{1}_{\alpha_{1}, \alpha_{2}}$, $\forall n \in \mathbb{N}$. To establish the converse, let $g_{n}$ be in the range of $\mathbf{l}_{\alpha_{1}, \alpha_{2}}, \forall n \in \mathbb{N}$. Then there is $\phi_{n} \in \digamma_{\text {loc }}(0, \infty)$ such that $\mathbf{1}_{\alpha_{1}, \alpha_{2}} \phi_{n}=g_{n}, n \in \mathbb{N}$.

Since $\left[\frac{\left(g_{n}\right)}{\left(\psi_{n}\right)}\right] \in \boldsymbol{\alpha}\left(\digamma_{\text {loc }}, \Omega, *, \circledast\right)$ we get $g_{n} \circledast \psi_{m}=g_{m} \circledast \psi_{n}, \forall m, n \in \mathbb{N}$. Therefore, Theorem 3 yields

$$
\mathbf{1}_{\alpha_{1}, \alpha_{2}}\left(\phi_{n} * \delta_{m}\right)=\mathbf{1}_{\alpha_{1}, \alpha_{2}}\left(\phi_{m} * \delta_{n}\right), \quad \forall m, n \in \mathbb{N},
$$

where $\phi_{n} \in \digamma_{\text {loc }}(0, \infty)$ and $\left(\delta_{n}\right) \in \Delta, \forall n \in \mathbb{N}$. Thus $\phi_{n} * \delta_{m}=\phi_{m} * \delta_{n}, m, \forall n \in \mathbb{N}$. Hence,

$$
\left[\frac{\left(\phi_{n}\right)}{\left(\delta_{n}\right)}\right] \in \boldsymbol{\alpha}\left(\digamma_{\mathrm{loc}}, \Omega, *, *\right) \quad \text { and } \quad \mathbf{1}_{\alpha_{1}, \alpha_{2}}^{g e}\left(\left[\frac{\left(\phi_{n}\right)}{\left(\delta_{n}\right)}\right]\right)=\left[\frac{\left(g_{n}\right)}{\left(\psi_{n}\right)}\right] \text {. }
$$

The theorem is therefore completely proved. 
Theorem 16 The mappings $\mathbf{l}_{\alpha_{1}, \alpha_{2}}^{g e}$ are continuous with respect to $\delta$ and $\Delta$-convergence.

The proof of this theorem is given by various papers of many authors. A detailed proof is therefore omitted.

\section{Concluding remark}

The present study defined the space of Boehmians for some Meijer type integral transform and desired some properties in the class of Boehmians. The results given in this paper are seen to generalize those given by Roopkumar in $[15,23]$.

\section{Competing interests}

The authors declare that they have no competing interests.

\section{Authors' contributions}

All authors read and approved the final manuscript.

\section{Author details}

${ }^{1}$ Department of Mathematics, Anand International College of Engineering, Jaipur, 303012, India. ${ }^{2}$ Department of Applied Sciences, Faculty of Engineering Technology, Al-Balqa' Applied University, Amman, 11134, Jordan. ${ }^{3}$ Department of Mathematics, Hanseo University, Chungnam-do, Seosan-si, 356-706, Republic of Korea.

\section{Acknowledgements}

Authors of this article express their thanks and gratitude to the referees for their valuable comments and suggestions that improved the present article.

Received: 5 October 2015 Accepted: 1 February 2016 Published online: 19 February 2016

\section{References}

1. Meijer, CS: Eine nene erweiterung der Laplace transformation. Ned. Akad. Wet. Proc. Ser. A 44, 727-739 (1941)

2. Pandey, RN: A generalization of Meijer transform. Proc. Camb. Philos. Soc. 67, 339-345 (1970)

3. Srivastava, HM: A relation between Meijer and generalized Hankel transforms. Math. Jpn. 11, 1-13 (1966)

4. Srivastava, HM, Vyas, OD: A theorem relating generalized Hankel and Whittaker transforms. Ned. Akad. Wet. Proc. Ser. A 72(2), 1-13 (1969)

5. Krätzel, E: Integral transformation of Bessel-type. In: Proceed. Internat. Conf. General. Funct. Operati. Calculus, Varna, pp. 148-155 (1975)

6. Krätzel, E: Bemerkunger zur Meljer - transformatlon und anwendungen. Math. Nachr. 30, 327-334 (1965)

7. Krätzel, E: Eine Verallgemeinerung der Laplace und Meljer transformation. Wiss. Z. Univ. Jena, Math. Naturw. Reihe. Heft. 5, 369-381 (1965)

8. Krätzel, E: Die faltung der L-transformation. Wiss. Z. Univ. Jena, Math. Naturw. Reihe. Heft. 5, 383-390 (1965)

9. Waphare, BB: Meijer type transformation and related results. Int. J. Phys. Chem. Math. Fundam. 4(1), 7-16 (2013)

10. Rodriguez, J: A new variant for the Meijer's integral transform. Comment. Math. Univ. Carol. 31(3), $543-555$ (1990)

11. Conlan, C, Koh, SL: On the Meijer transformation. Int. J. Math. Math. Sci. 1, 145-159 (1978)

12. Rodriguez, J: Sobre una variante de la K-transformation. In: Actas V Congreso de Ecuaciones Diferenciales y Aplicaciones, Tenerife, pp. 503-513 (1982)

13. Betancor, JJ: On a variant of the Meijer integral transformation. Port. Math. 45(3), 251-264 (1988)

14. Boehme, TK: The support of Mikusinski operators. Trans. Am. Math. Soc. 176, 319-334 (1973)

15. Karunakaran, V, Roopkumar, R: Operational calculus and Fourier transform on Boehmians. Colloq. Math. 102, 21-32 (2005)

16. Karunakaran, V, Vembu, R: Hilbert transform on periodic Boehmians. Houst. J. Math. 29, 439-454 (2003)

17. Mikusinski, P: Fourier transform for integrable Boehmians. Rocky Mt. J. Math. 17, 577-582 (1987)

18. Al-Omari, SKQ: On a generalized Meijer-Laplace transforms of Fox function type kernels and their extension to a class of Boehmians. Georgian Math. J. (2015, in press)

19. Al-Omari, SKQ: Some characteristics of S transforms in a class of rapidly decreasing Boehmians. J. Pseud.-Differ. Oper. Appl. 5(4), 527-537 (2014)

20. Al-Omari, SKQ: Hartley transforms on a certain space of generalized functions. Georgian Math. J. 20(30), 415-426 (2013)

21. Al-Omari, SKQ, Kilicman, A: On generalized Hartley-Hilbert and Fourier-Hilbert transforms. Adv. Differ. Equ. 2012, 232 (2012). doi:10.1186/1687-1847-2012-232

22. Nemzer, D: Periodic Boehmians. Int. J. Math. Math. Sci. 12, 685-692 (1989)

23. Roopkumar, R: Mellin transform for Boehmians. Bull. Inst. Math. Acad. Sin. 4(1), 75-96 (2009) 